\title{
Joint Passive Time Reversal and Multichannel Equalization for Underwater Communications
}

\author{
João Gomes \\ Instituto Superior Técnico \\ Instituto de Sistemas e Robótica \\ Av. Rovisco Pais, 1049-001 Lisboa, Portugal \\ jpg@isr.ist.utl.pt
}

\author{
António Silva, Sérgio Jesus \\ SiPLAB - Universidade do Algarve \\ Campus de Gambelas, 8005-139 Faro, Portugal \\ asilva@ualg.pt, sjesus@ualg.pt
}

\begin{abstract}
Time reversal has aroused considerable interest in communications applications as a means of partially compensating for the intersymbol interference introduced by the channel. This paper presents experimental results for a passive time reversal experiment conducted off the west coast of Portugal during the MREA'2004 mission. A single acoustic projector generated a 2/4-PSK stream at 200 and 400 baud, modulated around a carrier frequency of $3600 \mathrm{~Hz}$. The signals were received at a range of about $2 \mathrm{Km}$ from the source on a sparse vertical array with 8 hydrophones. We examine several strategies for demodulating the data, including equalization, time-reversal, and combinations of both techniques. This work emphasizes the use of low-complexity multichannel combining algorithms for tracking channel variations prior to generating a passively focused signal.
\end{abstract}

\section{INTRODUCTION}

Time reversal — both active and passive [1] — has aroused considerable interest recently in communications applications as a means of partially compensating for the intersymbol interference (ISI) introduced by the channel [2].

Time reversal is a wave focusing technique that exploits the reciprocity of wave propagation in linear media to concentrate energy at a desired focal spot with no specific information about the environment. In its basic active form, time-reversed focusing is achieved by transmitting a channel probe from the intended focal spot to an array of transducers that sample the incoming pressure field. When these signals are played back in reverse they generate a reciprocal field that converges on the source location and approximately regenerates the original probe with low residual ISI. Due to its peculiar mode of operation, this type of source/receiver array is frequently referred to as a time-reversal mirror (TRM). In digital communications the retransmitted distorted probes modulate a communications waveform, which can then be demodulated at the focus with relatively low algorithmic complexity.

Passive time reversal is conceptually similar, yet both the probe and message are sequentially sent from the focal spot, so the array only operates in receive mode. Focusing is performed synthetically at the array by convolving the time-reversed distorted probes with the received data packet [1], [3]. This is in fact a multichannel combining (MC) strategy whose parameters are directly measured from the data, rather than derived by optimizing a cost function as in [4].
The results of several experiments at sea reported in the technical literature suggest that time reversal by itself will not ensure reliable detection of the transmitted symbols, and must be complemented by adaptive equalization at the receiver (see also the theoretical results of [5]). Nonetheless, this computationally inexpensive technique is useful because, when compared with full multichannel equalization, it allows simpler equalizers to be used. Arguably, the resulting overall reduction in complexity at the receiver more than makes up for the moderate degradation in performance.

In a few papers that have been published on simultaneous equalization and time reversal the two systems are operated in tandem, i.e., time reversal creates a single-channel signal which is then independently processed by an equalizer [6]. This configuration is inevitable in active time reversal, where the ocean itself generates the refocused waveform. In passive time reversal, however, the signals received at an array of hydrophones are synthetically combined, and may therefore be individually postprocessed after convolving them with estimates of the (time-reversed) channel impulse responses. We examine low-complexity approaches where a single combining coefficient is used per array sensor. These coefficients are adjusted at each symbol interval by either iteratively minimizing the output mean-square error (MSE) or maximizing the output magnitude. This approach is motivated by the observation that poor signal-to-interference (ISI+noise) ratio in synthetic focusing can often be attributed to destructive interference between contributions from different hydrophone signals, in spite of seemingly appropriate temporal alignment. Note that these are actually very short multichannel equalizers, and one could envisage using more elaborate filters as described in [4]. This, however, would drive up the algorithmic complexity and somewhat reduce the appeal of time reversal. Alternatively, it is possible to use lower-complexity single-channel equalizers to cancel some of the residual ISI in the focused signal.

This paper presents experimental results from a passive time-reversal experiment conducted off the west coast of Portugal during the MREA'2004 mission. A single acoustic projector generated a 2/4-PSK stream at 200 and 400 baud, and the signals were received at a range of about $2 \mathrm{Km}$ from the source on a vertical array with 8 unevenly-spaced hydrophones. In addition to equalization and channel char- 
acterization issues, we also briefly examine the impact of Doppler and probe estimation on performance.

\section{TIME REVERSAL}

This work emphasizes bandwidth-efficient coherent communications. As often done in this context, a complex representation in terms of lowpass equivalent signals will henceforth be adopted. Let $p(t)$ represent the basic pulse shape of the PAM sequences that are exchanged between the source and the TRM, which can also act as a convenient channel probe. Denoting by $g_{m}(t)$ the impulse response between the focal point and the $m$-th sensor of an $M$-element array, the distorted received probe is $h_{m}(t)=p(t) * g_{m}(t)$, where $*$ is the convolution operator. In passive phase conjugation the probe transmission is followed by a data packet after a guard interval, such that the received signal component at the $m$-th sensor is given by

$$
y_{m}(t)=\sum_{k} a(k) h_{m}\left(t-k T_{b}\right),
$$

where $a(k)$ denotes a symbol in the complex signal constellation and $T_{b}$ is the signaling interval. The output of a plain passive mirror is obtained by convolving the received packet with the time-reversed probe replica in each sensor and adding all contributions

$$
\begin{aligned}
z(t) & =\sum_{m=1}^{M} h_{m}^{*}(-t) * y_{m}(t) \\
& =\sum_{k} a(k) \sum_{m=1}^{M} h_{m}^{*}(-t) * h_{m}\left(t-k T_{b}\right) \\
& =\sum_{k} a(k) r\left(t-k T_{b}\right) * q(t) .
\end{aligned}
$$

In (2) the equivalent undistorted signaling pulse $r(t)=p(t) *$ $p^{*}(-t)$ was defined, and $q(t)$ denotes the so-called $q$-function $(\mathrm{QF})$, which is the sum of the temporal autocorrelations of the medium impulse responses

$$
q(t)=\sum_{m=1}^{M} g_{m}^{*}(-t) * g_{m}(t)
$$

Multipath self-compensation in a TRM implies that $q(t)$ should be approximately constant across the bandwidth of $p(t)$ (and $r(t)$ ), so that $r(t) * q(t) \propto r(t)$ and an undistorted PAM waveform is regenerated. In practice a delay is introduced to ensure causality of the time-reversed probe in (2), and all operations are performed in $L$-oversampled discrete-time signals $y_{m}(n) \triangleq y_{m}\left(n T_{b} / L\right)$ and $h_{m}(n)$.

Decoding is particularly simple when $p(t)$ has a root raised-cosine shape because then $r(t)$ is a Nyquist pulse. As discussed in [3], out-of-band noise removal can be accomplished by actually transmitting root-root raised-cosine signaling pulses $s(t)$ such that $p(t)=s^{*}(-t) * s(t)$, and then prefiltering all received waveforms by $s^{*}(-t)$ to reject noise and attain the desired equivalent pulse shape $p(t)$.

\section{A. Synchronization and Normalization}

a) Symbol Synchronization: If ideal regeneration were attained for a PAM signal built from Nyquist pulses, the TRM output should be sampled at $t=n T_{b}$ and the sequence of decision variables applied to a memoryless slicer. In a practical TRM, however, the output should first undergo symbol synchronization to determine the time offset that maximizes a performance metric such as detection SNR. Because the Doppler compensation technique of Section II-B.2 virtually eliminates any discrepancies in symbol rate at the TRM, we simply calculate the $L$ polyphase components of the oversampled discrete-time output, $z^{(l)}(n) \triangleq z\left((l+n L) T_{b} / L\right)$, $l=0, \ldots, L-1$, and choose the one with strongest average power.

b) Phase Synchronization: Doppler compensation was found to be effective at stabilizing the output constellation, eliminating frequency mismatches that result in sustained rotation of constellation points over time. Still, the widelyused approach of [4] for MMSE phase synchronization with a PLL is needed at the TRM output to properly align the constellation and track slow phase variations. This technique can be used even if an arbitrary real scaling exists between the output and reference, as is the case here.

c) Output Normalization: The final operation to be performed after symbol synchronization and constellation alignment is to account for an unknown scaling introduced by the channel and amplifiers at the transmitter and TRM (e.g., due to propagation loss or AGC). To avoid obscuring the effect of channel variations on the performance analysis of Section V, we simply calculate the real scaling factor that minimizes the MSE between the symbol-spaced output and the reference on a block of symbols after the channel probes become available, and use it throughout the data packet. In a practical implementation this gain would be continuously tracked with a digital AGC.

\section{B. Coherence Issues}

The overall time-reversed pulse shape in (2) is a sum of $M$ contributions $r(t) * g_{m}^{*}(-t) * g_{m}(t)$, each having a main lobe at $t=0$ and typically several others at various delays due to multipath. TRM focusing ensures that the lobes at $t=0$ are reinforced when summing over $m$, while all others tend to cancel each other, resulting in a pulse that closely resembles $r(t)$. These pulses have conjugate symmetry and, in particular, take on real (positive) values at $t=0$. This is the key observation that motivates the MC algorithms proposed in Section III: All sensor contributions interfere constructively at $t=0$, thus maximizing the magnitude of the resulting pulse shape.

1) Probe Estimation: To reduce the latency and mismatch between probe measuring and focusing, it is possible to discard the actual probe transmission and estimate it directly from a known preamble in the data packet [6]. If done properly, this has the added benefit of reducing the additive noise component in the probe estimate $h_{m}(t)$, which generates undesirable convolutional noise during focusing according to 
(2). A similar idea was taken one step further in [7] and termed decision-directed passive phase conjugation (DDPPC). In DDPPC the channel is tracked throughout the data packet, using as reference either a known preamble (training sequence) or previous symbol decisions (decision-directed mode). This preserves a sharp QF even with very long data packets because a low-latency channel estimate is always available.

While [7] argues in favor of iterative block least-squares estimation, in this work we adopt the exponentially-windowed RLS algorithm for channel tracking, which has been extensively used as a tool in underwater channel equalization and identification.

2) Doppler Compensation: Doppler compression and expansion of waveforms arising from relative motion of the source and mirror can be modeled as just another type of channel time variation, but it is best to take advantage of the considerable underlying structure to compensate it.

Given a nominal transmitted passband waveform with carrier frequency $\omega_{c}, \tilde{x}(t)=\operatorname{Re}\left\{x(t) e^{j \omega_{c} t}\right\}$, the Doppler distorted replica observed over a single (instantaneous) path is

$$
\tilde{x}(t(1+\beta))=\operatorname{Re}\left\{x(t(1+\beta)) e^{j \omega_{c} \beta t} e^{j \omega_{c} t}\right\} .
$$

where $\beta$ is the time compression factor. In terms of baseband signals, this results in time compression of the original $x(t)$ and multiplication by a complex exponential with angular frequency $\omega_{c} \beta$. In a multipath environment several delayed contributions of the above type are observed, but if the propagation geometry and motion are predominantly horizontal all compression factors will be similar and compensating for the average Doppler suffices.

To estimate the time compression factor $\beta$ we buffer a full data packet, track the (truncated) channel response, and fit a first-order polynomial to the evolution of the delay of the strongest arrival. The factor $\beta$ is readily related to the slope of this line, but details are ommitted here for lack of space. Given $\beta$, the Doppler compensated received signal is obtained from $y_{m}(t)$ as

$$
y_{m}^{\prime}(t)=y_{m}\left(\frac{t}{1+\beta}\right) e^{-j \omega_{c} \frac{\beta t}{1+\beta}},
$$

and used in all subsequent time-reversal processing. The same Doppler correction is applied to received channel probes whenever they are available. In this work block resampling was performed in discrete time using the efficient resample Matlab function.

\section{Multichannel Combining}

The DDPPC approach mentioned in Section II-B.1 keeps the QF sharp by continuously tracking the channel response. While this operation is amenable to parallel processing, still the total number of parameters to estimate in a typical discrete tap-delay-line model of an underwater channel may be quite large and impose a significant computational burden, particularly when the RLS algorithm is used. Alternative strategies that perform simpler adaptation and require fewer parameters may therefore be of interest.
We propose a modification to the plain mirror (2) where a single adaptive coefficient $w_{m}$ multiplies each sensor contribution before summing to obtain the focused signal

$$
z(t)=\sum_{m=1}^{M} w_{m} z_{m}(t), \quad z_{m}(t)=h_{m}^{*}(-t) * y_{m}(t) .
$$

As explained in Section II-B the rationale for this structure is the observation that, for moderate mismatch, the constructive interference of pulse contributions at $t=0$ is partially lost even though their individual shapes are not severely affected. Under those circumstances, it should be possible to mitigate this effect by multiplying each one by an appropriately chosen coefficient $w_{m}$ to enhance the coherence. Below we examine four algorithms:

\section{A. Maximum Magnitude (MMAG)}

Each coefficient performs a pure phase rotation, $w_{m}=$ $e^{-j \theta_{m}}$, and the phases $\theta_{m}$ are chosen to maximize the expected square magnitude of the mirror output sampled at time $t=$ $n T_{b}$, denoted $z(n)$. The first sensor is arbitrarily chosen as a reference by setting $w_{1}=1$. The cost function is

$$
J_{\text {mag }}=E\left\{\left|z_{1}(n)+\sum_{m=2}^{M} z_{m}(n) e^{-j \theta_{m}}\right|^{2}\right\} .
$$

Differentiating with respect to $\theta_{m}$ yields

$$
\frac{d J}{d \theta_{m}}=2 \operatorname{Im}\left\{E\left\{\left(z(n)-z_{m}(n) e^{-j \theta_{m}}\right)^{*} z_{m}(n)\right\} e^{-j \theta_{m}}\right\}
$$

A PLL-type [4] adaptation rule for $\theta_{m}$ is obtained by ignoring the statistical expectation and using (8) as an error signal driving a loop filter. If a simple feedback gain $K$ is used, which is an appropriate choice when Doppler compensation is employed, the following rule results

$$
\begin{aligned}
\theta_{m}(n+1) & =\theta_{m}(n)+K \Phi_{m}(n), \\
\Phi_{m}(n) & =\operatorname{Im}\left\{\left(z(n)-z_{m}(n) e^{-j \theta_{m}}\right)^{*} z_{m}(n) e^{-j \theta_{m}}\right\} .
\end{aligned}
$$

\section{B. Constrained Minimum MSE (CMMSE)}

Unit-magnitude coefficients $e^{-j \theta_{m}}$ are used to minimize the cost function

$$
J_{m s e}=E\left\{\left|C a(n)-\sum_{m=1}^{M} z_{m}(n) e^{-j \theta_{m}}\right|^{2}\right\} .
$$

In (11) $C$ is a positive constant that reflects the fact that the TRM output approximates the original waveform up to a gain that depends on the channel and any scaling operation introduced in the processing chain. Now, if $|a(n)|=1$ and $C<\sum_{m}\left|z_{m}(n)\right|$, then the factors $z_{m}(n) e^{-j \phi_{m}}$ in (11) cannot all be aligned in phase because this would produce an output with magnitude greater than $C|a(n)|$, which does not minimize the MSE. Therefore, to attain full constructive interference the constant $C$ should be chosen such that $C \geq \sum_{m}\left|z_{m}(n)\right|$ for constellations where $|a(n)| \leq 1$. In principle, any value of $C$ greater than the lower bound above would be acceptable, but 


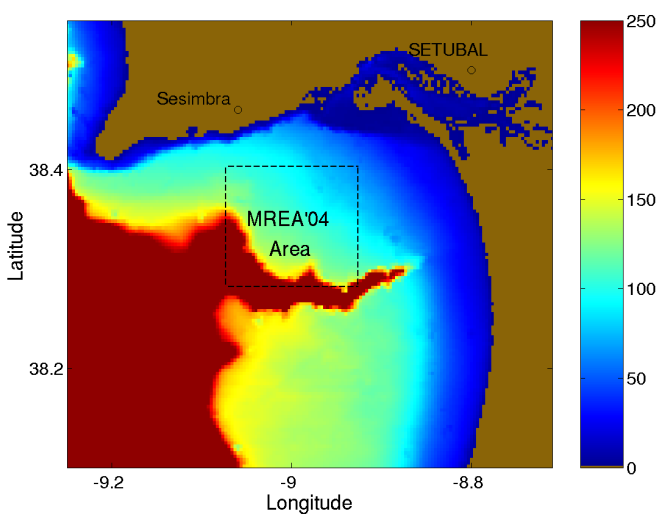

Figure 1: MREA'2004 test site

we chose to set it only $10 \%$ higher than the lower bound. That safety margin is enough to accomodate a moderate increase in received power due to channel variations during a single packet.

Replicating the steps in Section III-A yields an adaptation rule that is identical to (9), but driven by the error

$\Phi_{m}(n)=\operatorname{Im}\left\{\left(C a(n)-z(n)+z_{m}(n) e^{-j \theta_{m}}\right)^{*} z_{m}(n) e^{-j \theta_{m}}\right\}$.

This method aligns the output constellation as a byproduct, so subsequent phase synchronization as described in Section II-A becomes unnecessary.

\section{Unconstrained Minimum MSE (UMMSE)}

Rather than aligning the $z_{m}$ with unit magnitude rotations, arbitrary coefficients $w_{m}$ can be used to minimize the output MSE. This effectively constitutes a very simple multichannel equalizer with one tap per sensor, which exploits probe preprocessing to significantly reduce the number of parameters to track. In our work, time adaptation of the $w_{m}$ is carried out by the RLS algorithm. Not only does this method handle phase synchronization, as in CMMSE, but it also eliminates the need for output normalization.

\section{THE MREA'2004 EXPERIMENT}

The MREA'2004 (Maritime Rapid Environmental Assessment) sea trial was conducted off the west coast of Portugal in April 2004, in an area shown in Fig. 1. The site is located in the continental shelf to the north of the Setúbal Canyon, on water depths varying from 70 to $140 \mathrm{~m}$. The weather was calm during the acoustic trial, with sea state between 1 and 2, low wind of less than 10-15 knot, generally from the North quadrant, and wave height less than $2 \mathrm{~m}$.

The TRM is a vertical array with 8 hydrophones placed at depths $10,15,55,60,65,70,75,80 \mathrm{~m}$, installed on a drifting acoustic oceanographic buoy (AOB). The minimum sensor separation is about 12 wavelengths at the carrier frequency. The source was suspended from the support ship R/V ALLIANCE at depths ranging from 60 to $70 \mathrm{~m}$, depending on

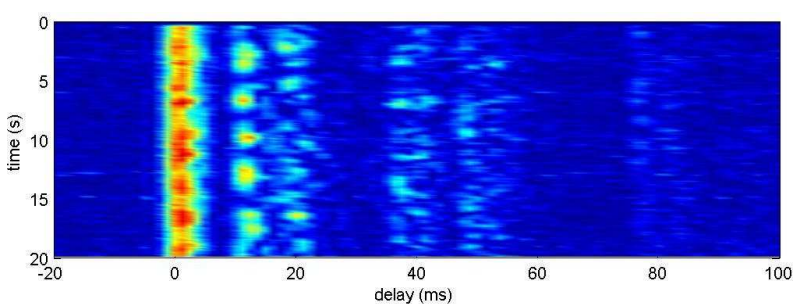

(a)

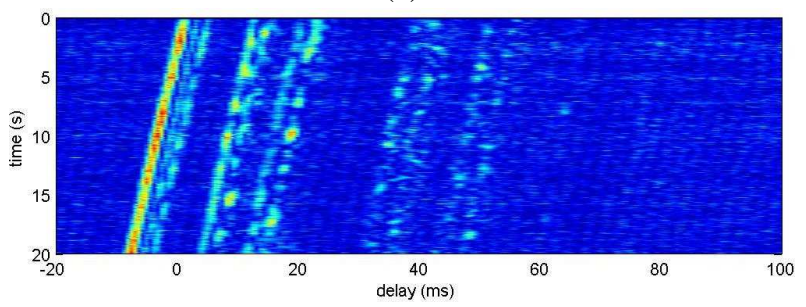

(b)

Figure 2: Evolution of estimated channel impulse responses before Doppler compensation (a) PKT 106 (200 baud, $f_{d o p}=$ $-0.11 \mathrm{~Hz}$ ) (b) PKT 149 (400 baud, $f_{d o p}=1.65 \mathrm{~Hz}$ )

speed. The experiment started at a close range of $0.6 \mathrm{Km}$ to the south of the AOB and the source progressively opened range to the southeast along an approximately range independent path (bottom depth $110 \mathrm{~m}$ ) up to $2 \mathrm{Km}$. Doppler shifts estimated from navigation data, no larger than about $2 \mathrm{~Hz}$ in magnitude, are consistent with those actually measured in acoustic signals.

During a period of approximately 90 minutes modulated data were transmitted at a carrier frequency of $3600 \mathrm{~Hz}$, using symbol rates of 200 or 400 baud, and both 2-PSK and 4-PSK constellations. As discussed in Section II, rootroot raised-cosine signaling pulses with $100 \%$ rolloff were used to simplify out-of-band noise removal at the receiver by matched filtering to the transmitted pulse shape. Each individual transmission comprises a single truncated signaling pulse acting as a channel probe with symmetrical guard intervals for a total duration of $1 \mathrm{~s}$, followed by a $20 \mathrm{~s}$ data packet. To improve the SNR when directly measuring channel responses, probe pulses were sent with double the amplitude of signaling pulses in data packets.

\section{EXPERIMENTAL RESUlts}

a) Channel Responses: Fig. 2 shows the evolution of estimated impulse responses at the top hydrophone in two of the received packets. The estimates were obtained by directly sampling the received signal at $L=4$ samples per symbol, and then using the polyphase components $y_{m}^{(l)}(n)=y_{m}(l+n L)$ as references to a bank ${ }^{1}$ of $L$ parallel exponentially-windowed RLS transversal filters fed by the known symbol sequence.

${ }^{1}$ Due to the cyclostationarity of PAM waveforms, MMSE channel identification using $L$-oversampled signals is equivalent to solving $L$ scalar and symbol-rate-sampled identification problems with fewer (by a factor of $L$ ) coefficients. This turns out to be computationally advantageous due to the quadratic increase in complexity of RLS as a function of the number of parameters to adapt. 


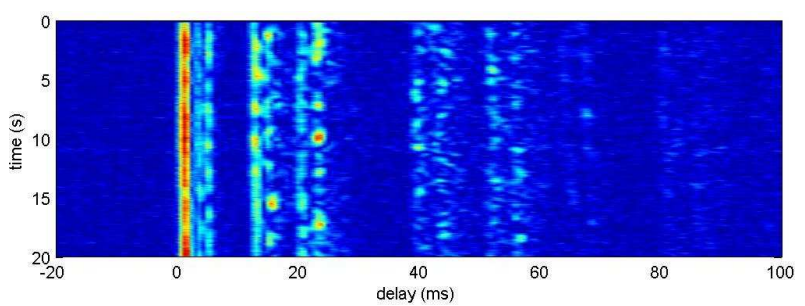

Figure 3: Channel impulse response of PKT 149 after Doppler compensation

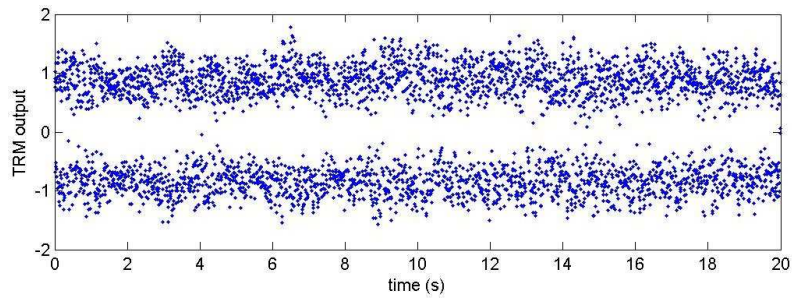

(a)

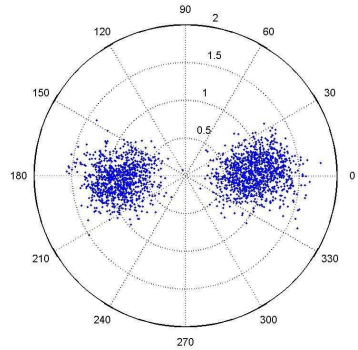

(b)

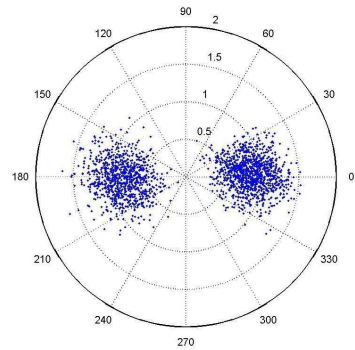

(c)
Figure 4: Plain TRM in PKT 106 (a) Time evolution with observed probes (b) Output constellation (c) Output constellation with estimated probes

Each of them operates with 40 causal and 10 anti-causal coefficients, and a forgetting factor $\lambda=0.9$ that was adjusted by trial and error to minimize the residual error variance. Snapshots of the coefficient vectors (estimated impulse responses) were taken every 20 symbol intervals and rearranged in the correct temporal order to produce the plots. The multipath arrival structure, spanning about $50 \mathrm{~ms}$, is clearly visible, as well as a time compression in Fig. $2 b$ due to Doppler that causes the main arrival to slip by 14 samples ( 3.5 symbols) in the course of a $20 \mathrm{~s}$ packet. Fig. 3 shows the impulse response estimate for the same packet after Doppler compensation as described in Section II-B.2.

b) Plain Time Reversal: Fig. 4 shows the plain TRM output for the packet pertaining to Fig. 2a. The upper plot shows the remarkably stable behaviour of $\operatorname{Re}\{z(n)\}$, generated from observed probes, over the packet duration. Figs. $2 \mathrm{~b}-\mathrm{c}$ show the output constellation with observed (TRMo) and estimated probes (TRMe). Performance was found to be similarly stable in several other processed packets, which is somewhat unexpected given that the source was moving, albeit at speeds not exceeding about $0.5 \mathrm{~m} / \mathrm{s}$. In the case of Fig. 3 the Doppler-compensated channel still exhibits significant

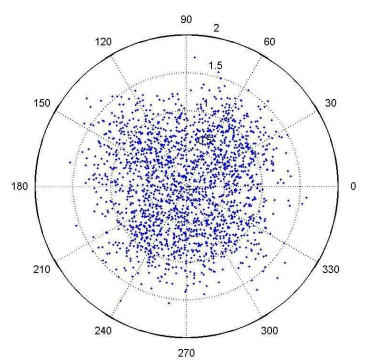

(a)

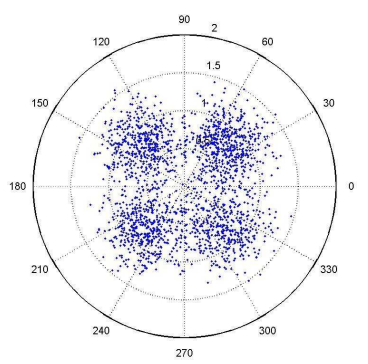

(b)
Figure 5: Plain TRM in PKT 149 (a) Output constellation with observed probe (b) Output constellation with estimated probe

variations, and due to the latency of probe observation this leads to a degradation in performace when compared with probe estimation (Fig. 5). Table I provides short-, mediumand long-term MSE estimates in some other packets. MSE values were calculated on the following blocks of symbols:

- Short-term MSE (s): Symbols 401-900

- Medium-term MSE (m): Symbols 901-1900

- Long-term MSE (l): Symbols 2401-3400

The output normalization factor introduced in Sec. II-A was calculated to minimize the MSE in the short-term block. RLS channel identification for probe estimation with oversampling factor $L=4$ was performed during the first 400 symbols. In all packets the observation interval or temporal support for impulse response estimation were chosen for best performance. They turn out to be considerably shorter than one would guess from the representative impulse responses in Figs. 2 and 3, typically about 4 or 5 symbol intervals.

Probe estimation tends to provide lower MSE values than probe observation, usually outperforming it by 0.5 to $1 \mathrm{~dB}$. Surprisingly, there is no clear dependency between output MSE and Doppler shift in the original data.

c) Multichannel Combining: Table II provides MSE values for joint time reversal using estimated probes and the simplified MC algorithms of Section III, as well as the DDPPC approach of [7] with impulse response snapshots taken every 10 symbol intervals. All PLL loop filters used the same feedback gain $K=0.05$. MMAG and CMMSE tend to provide a modest improvement in long-term MSE relative to plain time reversal, although they fail to handle medium- and long-term fluctuations in packets such as 25, where the channel varies considerably. Performance remains quite stable throughout the packet using DDPPC. In this case, increases in MSE over time are mainly due to misadjustment of the real gain used for output normalization, which is kept fixed. It is not surprising that UMMSE reaches lower MSE values than the other algorithms, as this is in fact a short multichannel equalizer. As in MMAG and CMMSE, where channel estimates are only computed once at the beginning of each packet, performance degrades over time.

d) Equalization: Table I also shows MSE values, averaged over whole packets, for TRMe followed by a singlechannel fractionally-spaced equalizer (FSE) operating at $L=$ 
Table I: Performance of plain time reversal and equalization

\begin{tabular}{|c|c|c|c|c|c|c|c|c|c|c|}
\hline \multirow{3}{*}{ PKT } & \multirow{3}{*}{ Baud/M-PSK } & \multirow{3}{*}{$f_{d o p}(\mathrm{~Hz})$} & \multirow{2}{*}{\multicolumn{3}{|c|}{$\begin{array}{c}\text { TRMo (obs. probes) } \\
\text { MSE (dB) }\end{array}$}} & \multirow{2}{*}{\multicolumn{3}{|c|}{$\begin{array}{c}\text { TRMe (estim. probes) } \\
\text { MSE (dB) }\end{array}$}} & \multirow{2}{*}{$\begin{array}{c}\text { TRMe+FSE } \\
\text { MSE (dB) }\end{array}$} & \multirow{2}{*}{$\begin{array}{c}\text { MFSE } \\
\text { MSE }(\mathrm{dB}) \\
\end{array}$} \\
\hline & & & & & & & & & & \\
\hline & & & $\mathrm{s}$ & $\mathrm{m}$ & 1 & $\mathrm{~s}$ & $\mathrm{~m}$ & 1 & & \\
\hline 25 & $200 / 2$ & -1.63 & -0.31 & 0.13 & -0.02 & -8.01 & -5.32 & 0.69 & -5.72 & -6.78 \\
\hline 106 & $200 / 2$ & -0.11 & -8.98 & -9.13 & -8.59 & -9.45 & -8.97 & -8.06 & -14.99 & -15.77 \\
\hline 141 & $400 / 2$ & -0.62 & -6.89 & -6.10 & -5.05 & -6.91 & -6.01 & -4.76 & -10.37 & -12.69 \\
\hline 149 & $400 / 4$ & 1.65 & -5.27 & -4.10 & -4.29 & -8.94 & -7.09 & -6.29 & -6.50 & -10.71 \\
\hline 153 & $200 / 2$ & 0.69 & -6.42 & -6.18 & -5.50 & -6.91 & -7.43 & -7.56 & -11.12 & -13.39 \\
\hline 186 & $200 / 2$ & -0.57 & -9.79 & -8.58 & -8.75 & -9.54 & -8.58 & -8.70 & -14.00 & -15.52 \\
\hline 200 & $400 / 4$ & -0.45 & -8.07 & -7.98 & -7.53 & -9.46 & -7.86 & -7.13 & -13.62 & -13.09 \\
\hline
\end{tabular}

Table II: MSE performance (in $\mathrm{dB}$ ) of joint time reversal and multichannel combining

\begin{tabular}{|c|c|c|c|c|c|c|c|c|c|c|c|c|}
\hline \hline & \multicolumn{3}{|c|}{ MMAG } & \multicolumn{3}{c|}{ CMMSE } & \multicolumn{3}{c|}{ UMMSE } & \multicolumn{3}{c|}{ DDPPC } \\
\hline PKT & $\mathrm{s}$ & $\mathrm{m}$ & $\mathrm{l}$ & $\mathrm{s}$ & $\mathrm{m}$ & $\mathrm{l}$ & $\mathrm{s}$ & $\mathrm{m}$ & $\mathrm{l}$ & $\mathrm{s}$ & $\mathrm{m}$ & 1 \\
\hline 25 & -7.87 & -5.32 & 0.97 & -7.07 & -3.24 & -0.13 & -10.88 & -8.05 & -4.26 & -8.19 & -7.31 & -7.79 \\
106 & -9.44 & -9.00 & -8.67 & -9.18 & -8.71 & -8.66 & -11.66 & -11.49 & -11.17 & -9.81 & -9.27 & -9.05 \\
141 & -7.13 & -6.10 & -4.79 & -7.46 & -6.35 & -5.77 & -7.76 & -7.81 & -7.23 & -7.60 & -6.95 & -5.73 \\
149 & -8.85 & -7.26 & -6.80 & -9.03 & -7.34 & -7.23 & -9.76 & -8.40 & -8.13 & -7.69 & -6.73 & -6.19 \\
153 & -7.49 & -7.95 & -8.47 & -7.33 & -8.07 & -8.50 & -9.87 & -10.05 & -10.52 & -7.78 & -7.14 & -7.17 \\
186 & -9.64 & -9.66 & -9.26 & -9.83 & -10.11 & -9.39 & -11.42 & -12.27 & -11.77 & -9.19 & -8.58 & -7.88 \\
200 & -9.97 & -8.37 & -7.57 & -10.01 & -8.74 & -8.04 & -11.18 & -10.38 & -9.52 & -8.09 & -7.77 & -7.10 \\
\hline \hline
\end{tabular}

2 samples per symbol and spanning 16 causal symbol intervals and 8 noncausal ones. This gives an indication of the extent of residual ISI on the focused signal generated by TRMe, and the amount of postprocessing that is needed to compensate it in a communication system.

Finally, MSE values are given in the table for the best linear processor $^{2}$, a multichannel FSE directly operating on the 8 hydrophone signals oversampled by $L=2$ and spanning 2 causal symbol intervals and 1 noncausal one. This should be interpreted as a practical lower bound on the achievable MSE with higher computational complexity, and shows the performance degradation incurred by simpler TRM-based schemes.

\section{CONCLUSION}

Experimental results were given for time-reversed demodulation of PSK data collected during the MREA'2004 mission. Several receiver architectures were examined, namely, plain TRM, TRM followed by FSE, mixed TRM/MC methods, DDPPC and MFSE. By itself, TRM incurs a significant performance penalty relative to multichannel equalizers (MFSE), and postprocessing is therefore required to lower the MSE. This can be accomplished either with a conventional single-channel equalizer or with joint unconstrained combining (UMMSE). Computational savings of TRM-based schemes relative to MFSE were not dramatic because the latter was able to operate over a very short time span, but the differences would probably have been greater at higher data rates. MC methods aimed at enhancing the coherence of time reversal with probe mismatch

\footnotetext{
${ }^{2}$ Multichannel DFE performance, not shown in Table I, was found to be very similar.
}

(MMAG, CMMSE) provided modest improvements, partly due to the exceptional stability of plain TRM in many of the packets.

\section{ACKNOWLEDGMENT}

This work was supported by Fundação para a Ciência e a Tecnologia (ISR/IST plurianual funding) through the POSConhecimento Program that includes FEDER funds.

\section{REFERENCES}

[1] D. R. Dowling, "Acoustic pulse compression using passive phaseconjugate processing," J. Acoust. Soc. Am., vol. 95, no. 3, pp. 1450-1458, Mar. 1994.

[2] G. F. Edelmann et al., "An initial demonstration of underwater acoustic communication using time reversal," IEEE J. Oceanic Eng., vol. 27, no. 3, pp. 602-609, July 2002.

[3] A. Silva et al., "Underwater acoustic communication using a "virtual" electronic time-reversal mirror approach," in Proc. V European Conf. on Underwater Acoust. (ECUA'O0), Lyon, France, June 2000.

[4] M. Stojanovic, J. A. Catipovic, and J. G. Proakis, "Adaptive multichannel combining and equalization for underwater acoustic communications," $J$. Acoust. Soc. Am., vol. 94, no. 3, pp. 1621-1631, Sept. 1993.

[5] M. Stojanovic, "Retrofocusing techniques for high rate acoustic communications," J. Acoust. Soc. Am., vol. 117, no. 3, pp. 1173-1185, Mar. 2005.

[6] J. Gomes and V. Barroso, "Asymmetric underwater acoustic communication using a time-reversal mirror," in Proceedings of MTS/IEEE OCEANS'O0, vol. 3, Providence, RI, Sept. 2000, pp. 1847-1851.

[7] J. A. Flynn et al., "Multichannel equalization by decision-directed passive phase conjugation: Experimental results," IEEE J. Oceanic Eng., vol. 29, no. 3, pp. 824-836, July 2004. 